\title{
Zusammenhang zwischen Parametern des Glukosetoleranztestes bei Jungbullen und deren Nachkommenzuchtwert
}

\author{
Herrn Professor Dr. D. Simon zum 70. Geburtstag gewidmet.
}

\section{Summary}

Title of the paper: Relationships between parameters of the glucose tolerance test by young sires and estimated their breeding values

A high milk performance connected to a sound health regarding metabolism and a sufficient fertility in dairy cows depends on a well balanced distribution of energy in body. The terms 'type of turnover' and 'type of deposition' embody two extremes of food energy preferably for milk production or for body composition. Insulin plays an outstanding role based on its central position in energetic metabolism. The function of insulin may be recorded by means of the intravenous glucose tolerance test (GTT). The reaction of insulin and glucose was investigated after infusion of $1 \mathrm{~g}$ Glucose $/ \mathrm{kg}^{0.75}$ because of the probable genetic determination of the reactive ability. The coefficients of heritability range from $h^{2}=0.16 \pm 0.10$ to $h^{2}=0.28 \pm 0.16$.

Investigating 28 sires the correlation coefficients amount to $r=0.5$ for parameters of gluccose tolerance test and estimated breeding values which is closer than those between pedigree breeding value and glucose tolerance test.This is expecting additional information for young sires before the insert of the test.

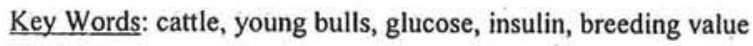

\section{Zusammenfassung}

Eine hohe Milchleistung bei stabiler Stoffwechselgesundheit und Fruchtbarkeit werden von einer ausgewogenen Energieverteilung im Körper beeinflußt. Das Insulin besitzt eine hervorragende Bedeutung für die Ausbildung der endokrin gesteuerten Stoffwechseleigenschaften. Die Reaktion von Insulin und Glukose waren nach Infusion von $1 \mathrm{~g}$ Glukose $/ \mathrm{kg}^{0,75}$ genetisch determiniert. Die Heritabilitătskoeffizienten rangieren von $\mathrm{h}^{2}=0,16 \pm 0,10$ bis $\mathrm{h}^{2}=0,28 \pm 0,16$. Der Zusammenhang zwischen Glukose-Toleranz-Test-Parametern und Nachkommenzuchtwert ist altersabhängig. Im 3. Lebenshalbjahr werden mittlere Korrelationskoeffizienten um $r=0,5$ erreicht, wăhrend sie zum Pedigrẹezuchtwert sehr lose sind. Dies läßt einen zusătzlichen Informationswert beim Jungbullen vor dem Testeinsatź erwarten.

Schlüsselwörter: Rind, Jungbullen, Glukose, Insulin, Zuchtwert

\section{Einleitung}

Eine hohe Milchleistung bei gleichzeitiger stabiler Stoffwechselgesundheit und ungestörtem Fruchtbarkeitsgeschehen der Milchkuh hängt in entscheidendem Maße von einer ausgewogenen Energieverteilung im Körper ab. Die verwendeten Begriffe des Umsatz- und Ansatztyps verkörpern zwei Extreme der Nutzung der zur Verfügung stehenden Nährstoffenergie vorrangig zur Milch- oder zur Körpermassebildung. Das Insulin besitzt aufgrund seiner zentralen Stellung im Energiestoffwechsel eine hervorragende Bedeutung für die Ausprägung der endokrin gesteuerten Stoffwechseleigen- 
schaften. Die Insulinfunktion kann mit Hilfe des intravenösen Glukosetoleranztestes (GTT) erfaßt werden. Ziel dieser Arbeit war es, die Wirkung verschiedener Einflußfaktoren auf die Ergebnisse des GTT zu untersuchen und einen Beitrag für die Aufklärung des Zusammenhangs zur Leistung zu erbringen.

\section{Material und Methoden}

Der intravenöse Glukosetoleranztest (GTT) wurde von BURKERT (1998) in 6 Aufzuchtstationen an insgesamt 411 Bullen der Rasse Deutsche Holsteins der Farbrichtungen Schwarzbunte $(n=329)$ und Rotbunte $(n=53)$ und der Rasse Angler $(n=29)$ mit einem Alter zwischen 129 und 2756 Tagen durchgeführt. Die Materialstruktur in den sechs Betrieben und Untersuchungsmonaten (Tab. 1) weist 411 Bullen mit 70 Wiederholungen aus. Bullen, die seit der letzten Fütterung am Nachmittag des Vortages lediglich Wasser aufnehmen konnten, wurde nach dem Legen einer verschließbaren Flexüle in die $V$. jugularis und der Gewinnung einer Ausgangsprobe zur Bestimmung der Basiswerte $1 \mathrm{~g}$ Glukose $/ \mathrm{kg}^{0,75}$ Körpermasse innerhalb von 1 bis 3 Minuten in Form einer 40 \%igen körperwarmen Glukoselösung injiziert. Nach der Glukoseinfusion wurden im Abstand von 7 Minuten 9 weitere Blutproben zur Erfassung der Insulinund Glukosereaktion gewonnen (Abb. 1).

Neben der Insulin-(I0) und der Glukosebasiskonzentration (G0) wurden für jedes Tier die maximalen Konzentrationswerte (IMAX1) und (GMAX1), die Glukosehalbwertzeit (GHWZ), die Insulin-(IA) und Glukoseflächen (GA) zwischen der jeweiligen Konzentrationsverlaufskurve und dem Basisspiegel sowie der Quotient der Flächenäquivalente IA/GA bestimmt. Auf der Basis der von BURKERT (1998) ermittelten Werte wurden in den vorliegenden Untersuchungen mit dem Programm PEST (GROENEVELD et al., 1993) für die einzelnen Parameter des GTT die Tiereffekte geschätzt. Die sowohl für die Ermittlung der Tiereffekte als auch für die Schätzung der Heritabilität erforderlichen Varianzkomponenten für die Faktoren 'Rest' und 'Tier' wurden mit dem Programm VCE 4.2 (GROENEVELD, 1998) berechnet. Die Schätzung erfolgte auf der Grundlage des Einmerkmalsmodells unter Berücksichtigung der Effekte der Rasse, der Herden-Probenahme und des Alters sowie des Tiereffektes. Die Schätzung erfolgte auf der Grundlage des folgenden Einmerkmalsmodells:

$$
y_{i j k l}=b_{i}+h p_{j}+a c_{k}+a_{i}+e_{i j k l}
$$

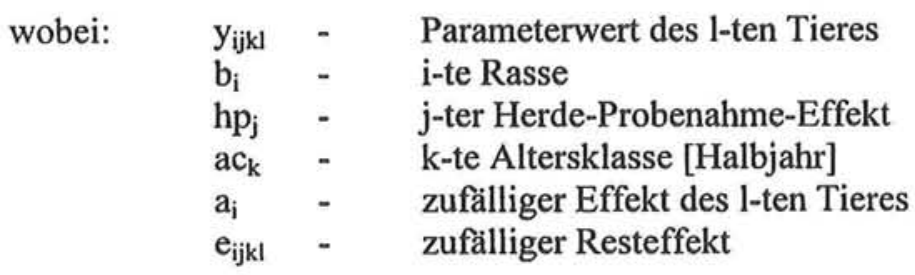




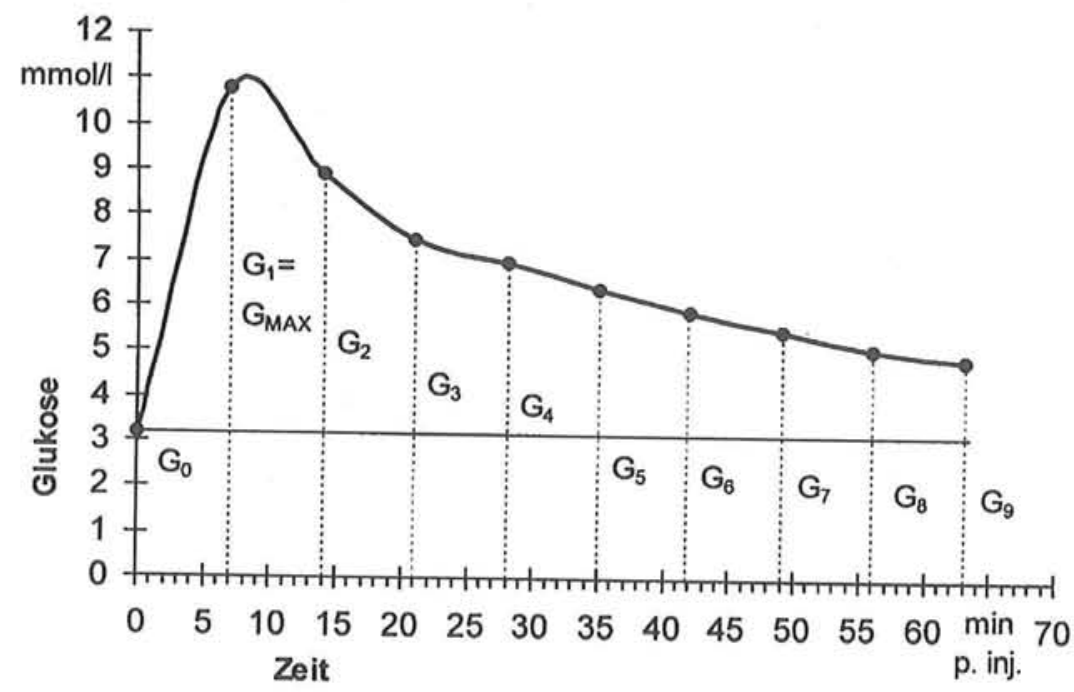

Charakterisierung der Glukoseantwort

$\mathrm{G}_{\mathrm{A}}=\mathrm{G}_{1}+\mathrm{G}_{2}+\ldots+\mathrm{G}_{9}-9 \times \mathrm{G}_{\mathrm{O}}$

$\mathrm{G}_{1}=\mathrm{G}_{\max }$

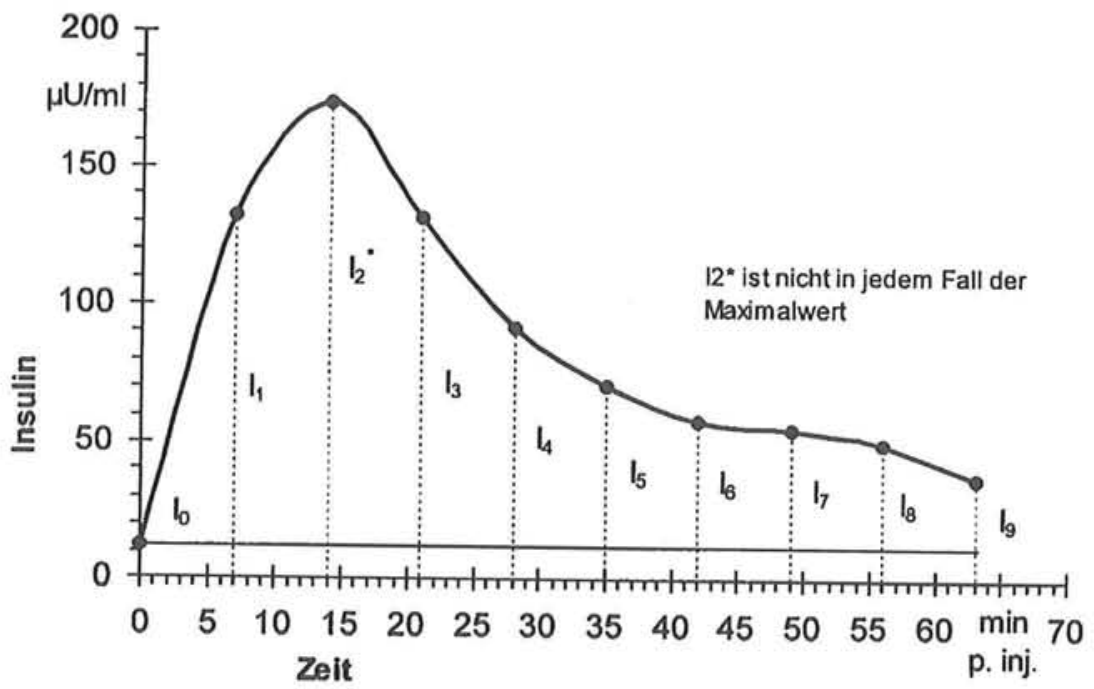

Charakterisierung der Insulinantwort

$\mathrm{I}_{\mathrm{A}}=\mathrm{I}_{1}+\mathrm{I}_{2}+\ldots+\mathrm{I}_{9}-9 \times \mathrm{I}_{0}$

$\mathrm{I}_{\mathrm{MAX}}=\mathrm{I}_{2}{ }^{\circ}$

Abb. 1: Charakterisierung der Glukose- und Insulinantwort (Reaction of glucose and insulin) 
Für insgesamt 282 schwarzbunte Bullen, die im GTT untersucht wurden, liegen auch Ergebnisse aus der Zuchtwertschätzung vor (Tab. 2). Die Verteilung der Bullen über die Betriebe und Lebenshalbjahre ist für die Untersuchung geeignet. Je Lebenshalbjahr werden 25 oder mehr Bullen vom zweiten bis 9. Lebenshalbjahr ausgewiesen. Zwischen den für diese Bullen ermittelten Tiereffekten aus den Parametern des GTT und den vom Informationssystem Tierhaltung Verden (VIT) veröffentlichten Nachkommen-Zuchtwerten werden korrelative Beziehungen mit SPSS berechnet. Alle Korrelationen werden innerhalb der schwarzbunten Bullen ausgewertet.

Tabelle 1

Verteilung der GTT auf die Probemonate und Betriebe (Investigation in months and herd)

\begin{tabular}{|c|c|c|c|c|c|c|}
\hline \multirow[t]{2}{*}{ Betrieb } & \multicolumn{5}{|c|}{ Monat } & \multirow[t]{2}{*}{$\Sigma$} \\
\hline & April & Mai & Juni & Oktober & November & \\
\hline 1 & 95 & & & & & 95 \\
\hline 2 & & 59 & 27 & & & 86 \\
\hline 3 & & & 49 & & 70 & 119 \\
\hline 4 & & & & 40 & & 40 \\
\hline 5 & & & 42 & 14 & & 56 \\
\hline 6 & 13 & 24 & 48 & & & 85 \\
\hline$\Sigma$ & 108 & 83 & 166 & 54 & 70 & 481 \\
\hline
\end{tabular}

Tabelle 2

Anzahl schwarzbunter Bullen mit Nachkommen-Zuchtwert (August 1999) nach Halbjahren und Betrieben (Bulls with estimated breeding values in life halb years)

\begin{tabular}{|c|c|c|c|c|c|c|c|}
\hline \multirow[t]{2}{*}{ Halbjahr } & \multicolumn{6}{|c|}{ Betrieb } & \multirow[t]{2}{*}{$\Sigma$} \\
\hline & 1 & 2 & 3 & 4 & 5 & 6 & \\
\hline 1 & & 6 & & $\because$ & & & 6 \\
\hline 2 & 6 & 18 & & & & & 24 \\
\hline 3 & 8 & 20 & & & & & 28 \\
\hline 4 & 12 & 1 & 1 & & 11 & & 25 \\
\hline 5 & 19 & 9 & 7 & 1 & 1 & & 37 \\
\hline 6 & 16 & 6 & 12 & 7 & 2 & 2 & 45 \\
\hline 7 & 12 & 4 & 7 & 8 & 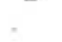 & 4 & 35 \\
\hline 8 & 10 & 5 & 5 & 7 & & 1 & 28 \\
\hline 9 & 4 & 3 & 3 & 8 & & 7 & 25 \\
\hline 10 & 1 & 7 & & 5 & & 3 & 16 \\
\hline$>10$ & 2 & 1 & 1 & 4 & & 5 & 13 \\
\hline$\Sigma$ & 90 & 80 & 36 & 40 & 14 & 22 & 282 \\
\hline
\end{tabular}

\section{Ergebnisse}

Zur Erzielung einer Normalverteilung wurden die Testparameter aus dem GTT logarithmiert (Tab. 3). Die ermittelten Heritabilitätskoeffizienten weisen auf einen genetischen Hintergrund der Parameter des Glukosetoleranztestes hin. Für die Parameter zur Erfassung der Glukosekonzentration und des Glukoseabbaues wurden Koeffizienten zwischen 0.16 und 0.28 geschätzt (Tab. 3). Die Meßwerte der Insulinantwort führten zu höheren Heritabilitätswerten im Bereich von 0,46 bis 0,53 , während für die Insulinbasiskonzentration keine genetische Determination nachgewiesen werden konnte. Für den Betrieb und die Probenahme konnte kein Einfluß mit Ausnahme des Betriebes 6 nachgewiesen werden. Daraus ist eine bedarfsgerechte Versorgung mit Futter abzulei- 
ten (BURKERT, 1998).

Die Glukosehalbwert (GHWZ) und das Insulinmaximum (IMAXI) verhalten sich in der Altersabhängigkeit gegenläufig. Sie erreichen im 3. Lebensjahr ihr Minimum bzw. Maximum (Abb. 2).

Tabelle 3

Logarithmierte Parameter des intravenösen Glukosetoleranztestes (GTT) (Parameters of GTT after logarithmic transformation)

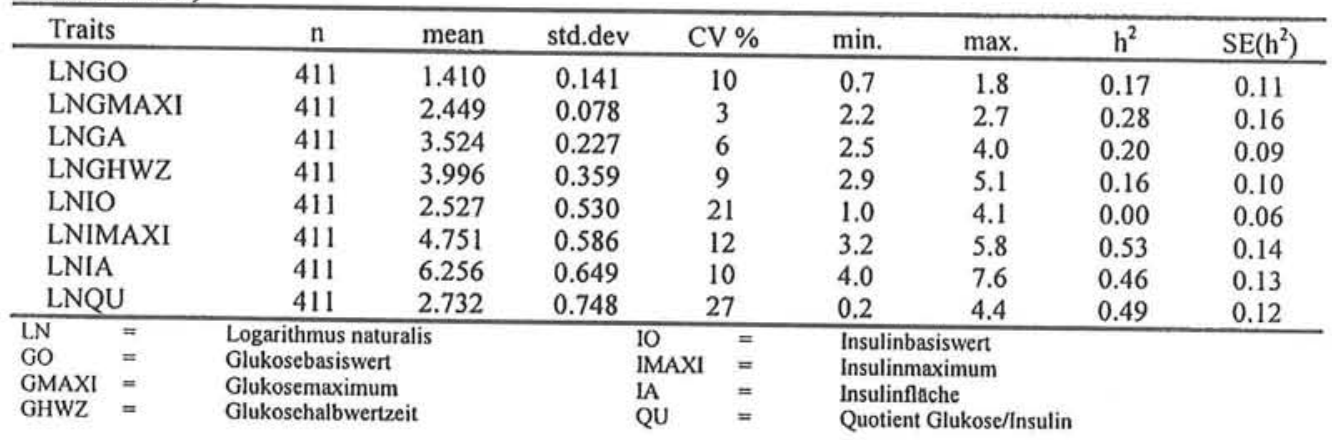

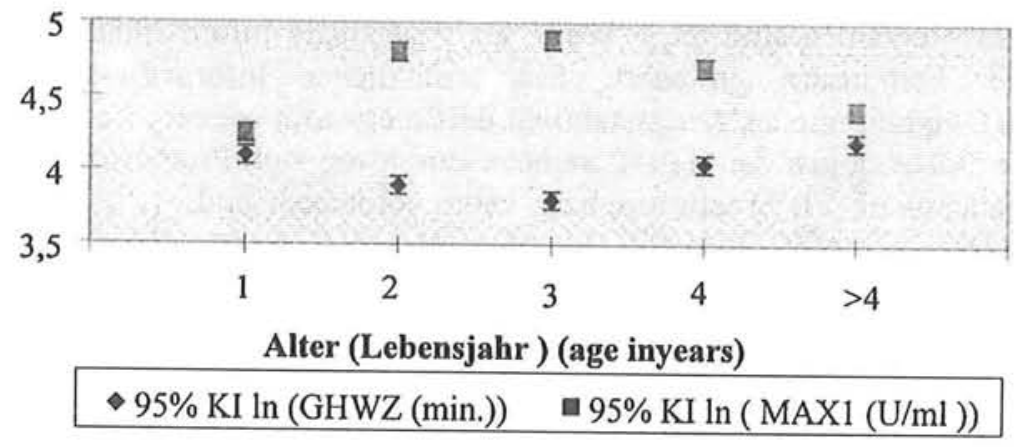

Abb. 2: Alterseinfluß auf die Glukose-Halbwertzeit (GHWZ) und das Insulin-Maximum (IMAXI) (Effect of age on the glucose halb life time (GHWZ) and the maximum of insulin (IMAXI))

Das Alter der Zuchtbullen zur Untersuchung nimmt wesentlichen Einfluß auf die Bewertung der einbezogenen Stabilitätsparameter im Glukosetoleranztest (GTT).

Die Beziehungen der Parameter des GTT am Bullen und seinem NachkommenZuchtwert sind in den Lebensabschnitten verschieden. Am Beispiel der Glukosehalbwertzeit (ln GHWZ, Tab. 4) ist zu erkennen, daß im dritten Lebenshalbjahr mittlere Korrelationskoeffizienten von $r=0,50(r=0,50$ bis $r=0,61)$ zu den Ertragsmerkmalen Milchmenge, Fett- und Eiweißmenge erreicht werden.

Ein Vergleich der Parameter des GTT untereinander in ihrer Beziehung zum Nachkommenzuchtwert in der Altersklasse 3 (3. Lebenshalbjahr, Tab. 5) zeigt, daß mit engeren Korrelationskoeffizienten die Glukose- den Insulinkennwerten überlegen sind. Dabei sind Glukosehalbwertzeit (GHWZ) und Glukosefläche (GA) abhängige Informationen. Beide liegen bei den Ertragsmerkmalen Fett-, Eiweißertrag und Relativzuchtwert Milch (RZM) über dem Informationswert der Vorfahrenleistung im Pedigreezuchtwert. 
Tabelle 4

Korrelationen zwischen dem GTT-Parameter (1n GHWZ) und den Nachkommen-Zuchtwerten schwarzbunter Bullen nach Lebenshalbjahren (ZW-Schätzung August 1999) (Correlations between the parameters intravenous glucose test (InGHWZ) and estimated breeding values)

\begin{tabular}{|c|c|c|c|c|c|}
\hline \multirow{2}{*}{$\begin{array}{l}\text { Lebens- } \\
\text { halbjahr }\end{array}$} & \multirow{2}{*}{$\begin{array}{c}\text { Anzahl } \\
\mathrm{n}\end{array}$} & \multicolumn{4}{|c|}{ Nachkommenzuchtwert (E B V) } \\
\hline & & Milchmenge & Fettmenge & Eiweißmenge & RZM \\
\hline 1 & 6 & $-0,22$ & $-0,08$ & $-0,15$ & $-0,12$ \\
\hline 2 & 24 & $-0,01$ & $-0,23$ & $-0,18$ & $-0,20$ \\
\hline 3 & 28 & $-0,61^{* *}$ & $-0,50^{\circ *}$ & $-0,52^{* *}$ & $-0,57^{* *}$ \\
\hline 4 & 25 & 0,35 & 0,25 & 0,24 & 0,27 \\
\hline 5 & 37 & $-0,22$ & $-0,33^{\circ}$ & $-0,16$ & $-0,21$ \\
\hline 6 & 45 & $-0,18$ & $-0,15$ & $-0,09$ & $-0,11$ \\
\hline 7 & 35 & 0,02 & 0,15 & 0,11 & 0,13 \\
\hline 8 & 28 & 0,13 & 0,16 & 0,15 & 0,15 \\
\hline 9 & 25 & 0,00 & $-0,26$ & 0,04 & $-0,04$ \\
\hline 10 & 16 & 0,23 & 0,37 & 0,22 & 0,28 \\
\hline$>10$ & 13 & $-0,29$ & 0,01 & $-0,11$ & $-0,07$ \\
\hline$\Sigma$ & 282 & $-0,10$ & $-0,10$ & $-0,05$ & $-0,06$ \\
\hline
\end{tabular}

Bei Zusammenfassung der beiden Altersklassen (Tab. 6) zwei und drei $(2 .+3$. Lebenshalbjahr) liegen die Beziehungen bei mittleren Korrelationskoeffizienten von $\mathrm{r}=0,40$ immer noch ähnlich im Informationswert des Pedigreezuchtwertes. Bei steigenden Tierzahlen sind diese Werte als zusätzliche Information beim Jungbullen vor dem Testeinsatz gesichert. Ein zusätzlicher Informationswert über die Leistungsfähigkeit und Leistungsstabilität dürfte erwartet werden, da in den Tabellen 5 und 6 die Beziehungen der GTT-Parameter durchweg zum Proteinertrag und RZM im Pedigreezuchtwert (PBV) sehr lose bzw. kaum vorhanden sind.

Tabelle 5

Korrelationen zwischen den GTT-Parametern Nachkommenzuchtwert (EBV) und Pedigreezuchtwert (PBV) in Altersklasse $3(n=28)$ (Correlations between the parameters of intravenous glucose test (GTT), values of sire including offspring performance (EBV) and pedigree breeding values (PBV) in the age claas; $n=28$ )

\begin{tabular}{|c|c|c|c|c|c|c|}
\hline \multirow{2}{*}{$\begin{array}{l}\text { GTT- } \\
\text { Parameter }\end{array}$} & \multicolumn{4}{|c|}{ Nachkommenzuchtwert } & \multicolumn{2}{|c|}{ Pedigree-ZW } \\
\hline & Milch-kg & Fett-kg & Eiweiß-kg & RZM & Eiweiß-kg & RZM \\
\hline LNGMAXI & $+0,14$ & $+0,08$ & $+0,11$ & 0,11 & 0,18 & 0,20 \\
\hline LNGA & $-0,42^{\circ}$ & $-0,40^{\circ}$ & $-0,28$ & $-0,33$ & $-0,03$ & $-0,22$ \\
\hline LNGHWZ & $-0,61 *$ & $-0,50$ & $-0,52^{* *}$ & $-0,57^{* *}$ & $-0,12$ & $-0,16$ \\
\hline LNIA & $+0,24$ & $+0,39^{\circ}$ & $+0,20$ & $+0,27$ & $-0,16$ & $-0,14$ \\
\hline LNQU & $+0,33$ & $+0,45^{* \bullet}$ & $+0,25$ & $+0,33$ & $-0,11$ & $-0,04$ \\
\hline Pedigree-ZW & $+0,71^{* *}$ & $+0,15^{4 \star A}$ & $+0,40^{\circ}$ & $+0,26$ & - & - \\
\hline
\end{tabular}

Tabelle 6

Korrelationen zwischen GTT-Parametem ,Nachkommenzuchtwert (EBV) und Pedigreezuchtwert (PBV) in Altersklasse $2+3(n=52)$ (Correlations between the parameters of intravenous glucose test (GTT), values of sire including offspring performance (EBV) and pedigree breeding values (PBV) in the age clas $2+3(n=52)$ )

\begin{tabular}{|c|c|c|c|c|c|c|}
\hline \multirow{2}{*}{$\begin{array}{l}\text { GTT- } \\
\text { Parameter }\end{array}$} & \multicolumn{4}{|c|}{ Nachkommenzuchtwert } & \multicolumn{2}{|c|}{ Pedigree-ZW } \\
\hline & Milch-kg & Fett-kg & Eiweiß-kg & RZM & Eiweiß-kg & RZM \\
\hline LNGMAXI & $+0,03$ & $+0,10$ & $+0,02$ & 0,01 & $-0,02$ & 0,14 \\
\hline LNGA & $-0,35^{*}$ & $-0,33^{\circ}$ & $-0,30^{\circ}$ & $-0,33^{\star *}$ & $-0,02$ & $-0,05$ \\
\hline LNGHWZ & $-0,40^{\circ *}$ & $-0,37^{* *}$ & $-0,39^{* *}$ & $-0,41^{\star *}$ & $-0,10$ & $-0,10$ \\
\hline LNIA & $+0,18$ & $+0,20^{\circ}$ & $+0,14$ & $+0,17$ & $-0,09$ & $-0,01$ \\
\hline LNQU & $+0,28^{\circ}$ & $+0,28^{\circ \cdots *}$ & $+0,22$ & $+0,26$ & $-0,08$ & 0,02 \\
\hline Pedigree-ZW & $+0,68^{* *}$ & $+0,36^{* *}$ & $+0,46^{\circ}$ & $+0,32^{\circ}$ & - & - \\
\hline
\end{tabular}




\section{Diskussion}

Bei zusammenfassender Betrachtung der Korrelationen (Tab. 5 und 6) sind die Glukosehalbwertzeit (GHWZ) und die Glukosefläche (GA) geeignete Stabilitätsparameter für die Ertragsleistung.

Auch ihre Wiederholbarkeiten liegen bei BURKERT (1998) zwischen $r=0,5$ bis $r=0,7$. Die Heritabilitätskoeffizienten der Glukoseparameter sind geringer als bei den Insulinparametern, die kritisch zu betrachten sind. Vergleichbare Ergebnisse liegen aus Holland von VAN DER WERF (1995) und Dänemark LØWENDAHL et al. (1995) von weiblichen und männlichen Rindern vor. Es kann erwartet werden, daß sich die Ergebnisse der Heritabilitätsschätzung bei einer größeren Anzahl junger Bullen bei reduzierten Fehlern verändern und stabilisieren. Die losen Beziehungen der GTTParameter zum Pedigreezuchtwert (PBV) lassen eine weitere zusätzliche wirksame und effiziente Information für die Nachkommenleistung des Jungbullen erwarten. Nach den Korrelationskoeffizienten zwischen GHWZ sowie GA und dem Nachkommenzuchtwert (EBV) ist der Informationswert dieser phänotypischen Parameter als indirekte Eigenleistungsprüung bei Jungbullen mit $r=0,3$ bis $r=0,5$ höher als der Pedigreezuchtwert für den zu erwartenden Nachkommen-zuchtwert des Jungbullen. Diese hohen Werte werden beim Insulin nicht erreicht, obwohl das Insulin die Glukosekonzentration steuert. Die gesteuerte Größe ist effizienter als eine der Steuergrößen. Die zusammenfassende Bewertung beider Informationen des Pedigreezuchtwertes mit den GTT-Parametern läßt eine erhöhte Genauigkeit der Einschätzung der Erwartungsleistung der Nachkommen auf ein Bestimmtheitsma $\beta$ um $B=0,4$ bis $B=0,5$ für die Ertragsmerkmale erwarten. Dafür ist eine spezielle statistische Aufbereitung zu erarbeiten, sobald ausreichend Material dafür verfügbar ist.

Die Beziehung der GTT-Parameter der Zuchtbullen zu ihrem Zuchtwert wird vom Alter zum Untersuchungszeitpunkt beeinflußt (Tab. 4). Erst bei Untersuchung jüngerer Bullen, auf deren Zuchtwert man lange warten muß, werden Korrelationskoeffizienten zwischen $r=0,30$ bis $r=0,50$ erreicht, während bei älteren Bullen keine gesicherten Beziehungen nachzuweisen sind. Altersabhängige Unterschiede im Zusammenhang physiologischer Indikatoren zum Zuchtwert fanden auch ROBINSON et al. (1994) bei Refütterung nach drei Fastentagen.

Die Ergebnisse von Untersuchungen an weiblichen Rindern von REINICKE (1993) sprechen für die präpubertalen Zeiträume. Die Ergebnisse über den Einfluß des präpubertalen Lebensabschnittes auf die Milch-, Reproduktions- und Wachstumsleistung (PANICKE et al., 1994) lassen sich histologisch durch die verstärkte Zelldifferenzierung für das sekretorische Drüsengewebe des Euters (FALCONER, 1980), für den Prolactin- und Wachstumshormonspiegel (PURCHAS et al., 1971; PRITCHARD et al., 1972; AMIR, 1974) und durch die Hormonkonzentration im präpubertalen Lebensabschnitt (DAY et al., 1986) begründen. Die Erwartungen an die Ergebnisse der im zweiten und dritten Lebenshalbjahr untersuchten Zuchtbullen werden durch die Ergebnisse von REINICKE et al. (1993) an 44 Kühen und deren Kälbern mit $r=0,41$ von STAUFENBIEL et al. (1999), PANICKE et al.(1988) und PANICKE (1991a, b) unterstützt. Diese Altersabhängigkeit der GTT-Parameter ist in Kenntnis der genetisch determinierten Zell- und Organdifferenzierung, insbesondere in den kritischen Entwicklungsphasen wie dem postnatalen und dem präpubertalen Zeitraum der Rinder, 
nachzuvollziehen. Eine weitere mögliche Ursache ist in nichtidentifizierten mDNAs zu sehen, die in gleichen Geweben bei verschiedenen Tieren unterschiedlichen Alters und damit unterschiedlicher Entwicklung oder in gleichen Geweben von Tieren aus verschiedenen Umwelten differenziert exprimiert werden. Damit wird bei Rindern für quantitative Merkmale eine mögliche umweltabhängige Genexpression einbezogen, gemessen und gewertet .

Mit den GTT-Parametern wird eine physiologisch genetisch gesteuerte phänotypische Merkmalsausprägung gemessen. Bei den multigen bedingten quantitativen Merkmalen wird somit die Summe der genetischen Effekte einschließlich ihrer Wechselwirkungen untereinander sowie ihrer Wechselwirkungen zur Umwelt bei der Genexpression bereits zum Meßzeitpunkt einbezogen. Diese umweltabhängige Genexpression findet für die ausgewiesenen quantitativen Merkmale Berücksichtigung. Damit kann mehr Zuchtprognose als durch eine einfache Altersjustierung erreicht werden. Wenn die bisher erzielten Ergebnisse sich bestätigen, könnte vor dem Testeinsatz der Prüfbullen eine Empfehlung zum Glucosetoleranztest gegeben werden, die in die Bewertung der Zuchtbullen einbezogen wird. Damit kann die Prüfkapazität erweitert oder es können die Prüfkosten gesenkt werden.

Abschließend wird geschlußfolgert:

- Die gegenläufige Glukose- und Insulinreaktion im GTT ist vom Alter abhängig.

- Der Proteinertrag ist ein geeigneter Stabilitätsparameter.

- Der Zusammenhang der GTT-Parameter zum Nachkommen-Zuchtwert ist altersabhängig.

- Im 3. Lebenshalbjahr werden Korrelationskoeffizienten um $r=-0,5$ für die Ertragsmerkmale erreicht.

- Die GTT-Parameter der Jungbullen erreichen höhere Korrelationskoeffizienten als die Pedigreezuchtwerte.

- Ihre Genauigkeit im Vergleich der Informationsquellen entspricht etwa dem Wert der Eigenleistung einer Kuh mit 3 Laktationen.

- Die umweltabhängige Genexpression wird mit einbezogen und genutzt.

- Es könnte eine zusätzliche Empfehlung durch den Glukosetoleranztest vor dem Testeinsatz des Prüfbullen gegeben werden.

\section{Literatur}

AMIR, S.:

Early breeding of dairy heifers - prospects and limitation. 25. Jahrestag. Eur. Vereinig. Tierzucht, Kopenhagen, 1974

BURKERT, $\mathrm{O}$ :

Untersuchungen zum intravenøsen und modifizierten Glukosetoleranztest bei Zuchtbullen. Freie Universităt Berlin, Diss., 1998

DAY, M.L. et al.:

Effect of restriction of dietary energy intake during the prepubertal period on secretion of luteinizing hormone and responsiveness of the pituitary. J. Animal Sci., Albany, N.Y. 62 (1986), 1641-1648

FALCONER, I.R..

Aspects of the biochemistry, physiology and endocrinology of lactation. Austr. J. biol. Sci. Melbourne 33 (1980) 1, 71-74 
GROENEVELD, E.:

VCE 4 - Version 4.2 Federal Research Center of Agriculture, Mariensee, 1998, Germany

GROENEVELD, E.; KOVAC, M.; WANG, T.:

PEST - Multivariate Prediction and Estimation. Department of Animal Sciences University of Illinois, 1993

LØVENDAL, P.:

Effect of selection for milk fat yield on insulin response to glucose tolerance test in prepubertal and pubertal RED dane calves. $48^{\text {th }}$ EAAP; Wien, 1997

LØVENDAL, P.; NIELSEN, M.; VAN DER WERF, J.H.J.:

Genetic parameters for glucose induced insulin release in juvenile dairy cattle. $46^{\text {th }}$ Annual Meeting of the EAAP, Prag, 1995

OLDENBROEK, J.K.; GALESLOOT, P.A.J.; POOL, M.H.; VAN DER WERF, J.H.J.:

Effects of selection for milk yield on feed intake and metabolism of heifers in early lactation. $48^{\text {th }}$. Annual Meeting of the EAAP, Wien, 1997

PANICKE, L.:

Breeding of young cattle and productivity of cows. 42. Jahrestagung der EVT, 1991 in Berlin, Vol. 2, $125, \mathrm{C} 46,1991 \mathrm{a}$

PANICKE, L.:

Wachstumsverlauf der Färse und Produktionsleistung der Kuh. Mh. Vet-Med., Jena 46 (1991b) 10, 636639

PANICKE, L.; MATTHES H.-D.; FREYER, G.:

Aktuelle Probleme der Gesundheit und des Wachstums bei Jungrindern. Internationales Symposium der Sudböhmischen Universitatt in Ceske Budejovice im Februar 1994, 16-18 und 51-56

PANICKE, L.; BIEMANN, J.; KADOW, U.:

Aufzuchtqualităt der Färse und Produktionsleistung der Kuh. Tierzucht, Berlin 42 (1988), 148-149

PRITCHARD, D.E. et al.:

Growth, mammary, reproductive and pituitary hormone characteristics of Holstein heifers fed extra grain and melingesholocatate. J. Dairy Sci., Champaign, III 55 (1972), 995-1004

PURCHAS, R.W. et al:

Some carcass quality and endocrine criteria of Holstein heifers fed melangestrol acetate. J. Animal Sci., Albany/N.Y. 324 (1971), 628-635

REINICKE, U.:

Der intravenöse und modifizierte Glukosetoleranztest beim Milchrind - Einflußfaktoren und Beziehungen zur Milchleistung. FU Berlin, Diss., 1993

ROBINSON, D.L.; HAMMOND, K.; MCDOWELL, G.H.:

Relationships between breeding values and physiological responses to fasting and refeeding in dairy bulls: update for young animals. J. Animal Breed. Genet. 111 (1994), 257-264

STAUFENBIEL, R.; REINICKE, U.; PANICKE, L.:

Zum Glukosetoleranztest beim Rind. 1.Mitt.: Beziehungen zum Laktationsabschnitt und zur Milchleistung. Arch.Tierz., Dummerstorf 42 (1999) 1, 45-56

VAN DER WERF, J.H.J.; LøVENDAL, P.:

Genetic Parameters for induced growth hormone secretion in dairy calves. ADSA meeting, abstract, 1995

Eingegangen: 15.11 .1999

Akzeptiert: 15.03 .2000

Anschriften der Verfasser

Prof. Dr. LOTHAR PANICKE

Forschungsinstitut für die Biologie

landwirtschaftlicher Nutztiere (FBN)

Wilhelm-Stahl-Allee 2

D-18196 Dummerstorf

Prof. Dr. RUDOLF STAUFENBIEL

Freie Universităt Berlin

Klinik für Klauentiere

Königsweg 65

D-14163 Berlin

Dr. ECKHARD FISCHER

Universităt Rostock

Fachbereich Agrarokologie

Justus-von-Liebig-Weg 6

FRIEDRICH REINHARDT

D-18059 Rostock

VIT Informationssysteme

Heideweg 1

D-27283 Verden/Aller 
Arch. Tierz., Dummerstorf 43 (2000) 3, 240

\title{
Buchbesprechung
}

\section{Krankheiten des Rindes}

\author{
GUSTAV ROSENBERGER unter Mitarbeit von GERRIT DIRKSEN, HANS GRÜNDER und MATTHAEUS \\ STÖBER
}

3. unveränderte Auflage, 1430 Seiten, 747 Abbildungen im Text und auf 28 farbigen Tafeln, 70 Übersichten, Blackwell Wissenschafts-Verlag, Berlin, 1994, ISBN 3-8263-3029-3, DM 148,-, , 8 1080,-, sFr 136,5o

Trotz großer Fortschritte im Kenntnisstand erfolgsorientierter Rinderhalter, prophylaxeorientierter Rinderproduktion, Regelungen zur Haltung, Medikamentenanwendung und des Tierschutzes, sieht sich der Tierarzt in der Rinderhaltung mit Therapienotwendigkeiten konfrontiert. Mit zunehmender Intensivierung und Leistungssteigerung erlangen auch Stoffwechsel- und Mangelkrankheiten oder Parasiteninvasionen immer großere praktische Bedeutung. Auch der Einsatz von Schädlingsbekämpfungsmitteln im intensiven Pflanzen- und Futterbau können Anlass zu mehr oder weniger schwerwiegenden Vergiftungen geben. Auf alle, durch Krankheit bedingten St $0-$ rungen beim Rind, will dieses in dritter Auflage erschienene umfangreiche Standardwerk eine Antwort geben.

Die im Buch zu besprechenden Krankheiten werden entsprechend ihren Ursachen aufgegliedert. Den großBten Umfang nehmen die Organkrankheiten d.h. die sogenannten „sporadischen“ Leiden der einzelnen Organe und Organsysteme mit weiterleitenden Hinweisen zu deren spezifischen Krankheiten ein. Aus der Gliederung zu diesem Teil seien u.a. nur die Hauptabschnitte zu Krankheiten des Haarkleides, des Lymphapparates, der Kreislauforgane, des Atmungs-, Verdauungs-, Harn- und Bewegungsapparates, der Klauen, des Bewegungsapparates, des Nervensystems und der Sinnesorgane genannt. In diesem, wie in spăteren Buchteilen, werden fur jede Krankheit jeweils das Wesen, Vorkommen, die Ursachen die Erscheinungen, Erkennung, der Verlauf und die mögliche Behandlung beschrieben. Angefugt sind jeweils z.T. sehr ausfulhrliche Literaturverzeichnisse, die jedoch auflagebedingt die jüngeren Arbeiten nicht enthalten können. Bei der Beschreibung der therapeutischen Methoden finden nicht nur die medikamentठ̋sen und diătetischen Maßnahmen, sondern, wenn erforderlich, auch die operativen Eingriffe Erwăhnung, so dass innere Medizin und Chirurgie sich im Rahmen dieser Biuatrik ergänzen.

Es folgen die weiteren Hauptabschnitte der Infektionskrankheiten, parasităre Krankheiten, Stoffwechselstörungen und Mangelkrankheiten sowie der relativ umfangreiche Teil der Vergiftungen. Therapeutische Verfahren und Arzneimittelgruppen, die zur Behandlung mehrerer Leiden angezeigt sind, finden sich alphabetisch geordnet als umfangreicher „Therapeutischer Index“ zusammengefasst im Schlussteil des Buches.

Das Buch ist klar, sehr detailliert und ubersichtlich gegliedert. Die große Anzahl Abbildungen und zahlreiche Übersichten unterstutzen den hohen Informationswert dieses in klarer Sprache geschriebenen, praxisrelevanten, sorgfältigen und erschø̈pfenden Buches. Gute Sachkenntnis paart sich hier mit hervorragender Literaturubersicht. Es ist ein durch die Paperbackform besonders preiswertes Standardwerk, das nicht nur fur den Tierarzt, den Studierenden der Tiermedizin, den Wissenschaftler und den anspruchsvollen Tierhalter von Bedeutung ist, sondern auch für den Pathologen, Mikrobiologen, Parasitologen, Toxikologen, Physiologen oder Biochemiker.

Fur den praktischen Tierarzt ist dieses Buch unverzichtbar und bedarf eigentlich keiner besonderen Empfehlung. 